\title{
VELCRO-INSPIRED SUPRAMOLECULAR SYSTEM FOR SILICA-RUBBER COUPLING
}

\author{
Rafal AnyszKa, ${ }^{1,2, *}$ KAROlina Beton, $^{2}$ Maja SzCZECHOWICZ, ${ }^{2}$ DARIUsz M. Bielinski, ${ }^{2}$ AnKe Blume ${ }^{1}$ \\ ${ }^{1}$ University of TWENTE, FACUlty OF ENGINEERING TECHNOLOGY, DEPARTMENT OF MECHANICS OF SOLIDS, SURFACES \\ \& Systems (MS3), Chair of ElASTOMER TECHNOLOGY \& ENGINEERING, ENSCHEDE, THE NETHERLANDS

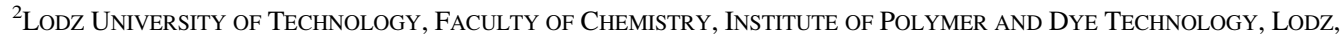 \\ POLAND
}

RUBBER CHEMISTRY AND TECHNOLOGY, Vol. 93, No. 4, pp. 672-682 (2020)

\begin{abstract}
The state-of-the-art silica-rubber coupling is based on forming chemical links between a silica surface and rubber macromolecules. However, the chemical links are relatively short and stiff, thus in case of a chemical breakage they are highly unlikely to recombine. This could result in a potential deterioration of the interphase properties over time. To overcome this drawback, a new approach to silica-rubber coupling was investigated in the current study. The new approach is inspired by the Velcro hook-and-loop system from nature that facilitates a re-connectability, thus re-formation of the interphase properties in case of a breakage. For this, various long oligomeric brushes were grafted onto silica surfaces considered to act as supramolecular hooks. Such modified silica were dispersed in rubber and vulcanized. The resulting cross-linked rubber matrix is considered to act as supramolecular loops. The prepared vulcanizates were compared with reference samples containing common coupling or covering agents. The reinforcing potential provided by the newly developed system is lower than the chemical coupling system but considerably higher than the covering system. The new system also provides better mechanical properties, recovery after cycling stretching, and heat treatment than the references. A new reinforcing mechanism is proposed for the silica grafted with oligomeric brushes that exhibits a good chemical compatibility to the rubber matrix. [doi:10.5254/rct.20.79966]
\end{abstract}

\section{INTRODUCTION}

The application of the silica-silane reinforcing system for rubber allows a significant improvement of wet grip and reduction of rolling resistance of a tire tread in comparison with carbon black. ${ }^{1}$ To achieve these improvements efficiently, coupling silane has to be used. The stateof-the-art silanes contain alkoxyl groups reactive with the silica surface via silanol groups. The surface modification follows a condensation reaction. After that, the di-, poly-sulfidic chains or thiol functional groups of the silane react with the unsaturated rubber chain during the vulcanization and thus form a strong chemical bond between the silica and rubber. However, in such a system, the sulfidic bond formed between the silica and rubber also exhibits relatively high stiffness, and if it breaks under external stress its re-formation is very unlikely. This could lead to a deterioration of silica-filler interfacial properties, thus decreasing the in-rubber performance.

To overcome this disadvantage, an inspiration for an alternative coupling system comes from nature. One of the most widely applied connecting systems mimicked from nature is the Velcro fastener. ${ }^{2}$ Velcro was invented by the Swiss engineer George de Mestral in 1941. The principle of the Velcro fastener is based on connecting two surfaces by a hook-and-loop system that facilitates easy re-connectability. However, currently it is applied only on micro-scale, in which the molecular mobility and miscibility is not crucial for the connection performance. The challenging task is to transfer this solution to nano-scale, which if implemented for silica-rubber coupling could significantly improve the reliability of the interphase properties in long term applications.

But is it feasible to effectively implement the Velcro approach on a supramolecular level to create a new silica-rubber interphase? A number of approaches were already introduced in the 
literature to establish re-connectable interactions between macromolecules of various polymers. However, none has been considered to implement this approach for polymer-filler interphase coupling. There are two main approaches to implement Velcro-like behavior on supramolecular level:

1. Host-guest (loop-hook) interactions: This concept is based on specific interactions between two functional moieties implemented in two different polymer phases. For example, Ahn et al. functionalized two surfaces with large cucurbit[7]uril groups (host/ loop) and aminomethylferrocene (guest/hook), resulting in strong adhesive bonds preserved even in an aqueous environment. ${ }^{3}$ Whereas, Siever et al. synthesized polymers with a grafted pyridine ring (guest/hook) and porphyrin zinc (II) complex (host/loop) that exhibits coordination reversible interactions. ${ }^{4}$ However, use of such a host-guest system for the silica-rubber coupling would require extensive modification of the rubber macromolecules to provide a sufficient amount of interacting groups. This would influence their mobility and could influence the dynamic properties, which leads possibly to an undesired increase in the rolling resistance of a tire. Moreover, the strong silicarubber interactions could result in a rise of the compound's viscosity, worsening its processing properties.

2. Macromolecular entanglements as possible physical joints coupling two surfaces that exhibit re-connectability due to their dynamics: This issue was addressed in 1993 by O'Connor and McLeish, who performed a model calculation of an end-tethered macromolecule diffusion into a cross-linked elastomer matrix. This study predicts a rapid partial toughness recovery of such interphase after a distortion, followed by much slower recovery to an equilibrium state. ${ }^{5}$ An example of such supramolecular behavior can be found in biological substrates exhibiting self-healing performance. ${ }^{6}$

Unlike the host-guest approach, the use of macromolecular entanglements as a physical coupling system exhibiting Velcro-like behavior for silica-rubber interphase seems to be more feasible. Therefore, it was chosen as the principle for this study by grafting long oligomeric chains on silica surface that act as molecular hooks, against the cross-linked rubber matrix (molecular loops).

In this paper a study of a Velcro-inspired silica-rubber system is presented. The influence of the grafted oligomer brushes on the in-rubber reinforcement is discussed from the point of view of the Payne effect of the rubber compounds and the mechanical properties of the vulcanizates. The newly developed coupling system is compared with the state-of-the art silica-silane coupling system (using bis(triethoxysilylpropyl)disulfide (TESPD)) and with a silica-silane covering system (using dodecyltriethoxysilane).

\section{EXPERIMENTAL TECHNIQUES}

The rubber compounds were prepared by $350 \mathrm{~cm}^{3}$ laboratory mixer (Brabender, Duisburg, Germany). Kinetics of vulcanization (temperature, $160^{\circ} \mathrm{C}$; oscillation, $1.67 \mathrm{~Hz}$; pressure, $4.49 \mathrm{bar}$ ) and Payne effect (strain sweep from 0.57 to $100 \%$, temperature $100{ }^{\circ} \mathrm{C}$ ) of the rubber compounds were measured with a TA Elite Rubber Process Analyser (TA Instruments, Leatherhead, UK). Cross-link density of the vulcanizates was investigated by the Flory-Rehner swelling method in toluene. The vulcanizates' tensile strength was measured by a universal testing machine Zwick Z05 (Zwick, Ulm, Germany) operation with the crosshead speed of $500 \mathrm{~mm} / \mathrm{min}$ according to ASTM D 412 standard. A hysteresis test was performed on Zwick Z05 (Zwick) by applying five cycles of elongation up to $300 \%$ of strain and recovery with the crosshead speed of $500 \mathrm{~mm} / \mathrm{min}$. To evaluate 


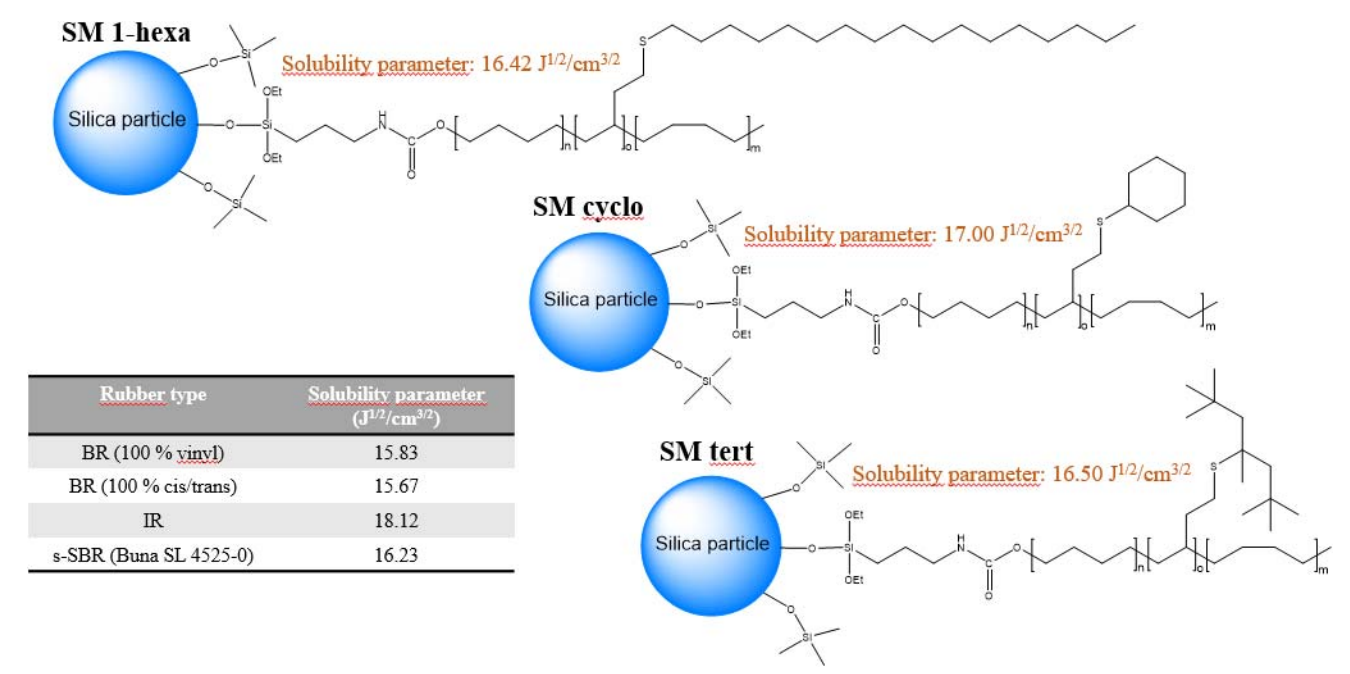

FIG. 1. - Chemical structures of the modified silica surfaces, with calculated solubility parameters of the grafted oligomers and of different rubber types.

the silica-rubber interactions recovery a thermal conditioning after the hysteresis was done in an air-circulating laboratory oven for $4 \mathrm{~h}$ at $80{ }^{\circ} \mathrm{C}$.

\section{MATERIALS}

Rubbers: Solution styrene-butadiene rubber (SSBR), Buna SL 4525-0 (Arlanxeo, Maastricht, the Netherlands); BR, Buna CB 24 (Trinseo, Schkopau, Germany). Silica: Zeosil 1165MP (Solvay, Beveren, Belgium). Coupling agent: bis(triethoxysilylpropyl) disulfide (TESPD) (Evonik, Essen, Germany). Covering agent: dodecyltriethoxysilane (Millipore Sigma, Darmstadt, Germany). Vulcanization activators: ZnO, stearic acid (Millipore Sigma). Antioxidants: N-(1,3dimethylbutyl)-N-phenyl-p-phenylenediamine (6PPD), poly(1,2-dihydro-2,2,4-trimethyl-quinoline (TMQ) (Caldic B.V., the Netherlands). Oil: Treated distilled aromatic extracted oil (TDAE) (Hansen \& Rosenthal, Hamburg, Germany). Curatives: Sulfur, diphenylguanidine (DPG), $N$-tertbutyl-benzothiazole sulfonamide (TBBS) (Caldic B.V., Nieuwegein, the Netherlands). Five types of pre-modified silicas were used for this study (four with long oligomer chains grafted on their surface and one modified with the covering agent as a reference), which modification procedure is described elsewhere. ${ }^{7}$

\section{METHODS}

The chemical structures of the grafted oligomers on the silica surface are presented in Figure 1, along with calculated solubility parameters. Vinyl groups of the oligomers were used to graft sidebranches using different thiols. This was done to improve the possible entanglement formation with the rubber macromolecules. One sample was not modified with thiols to be used as a reference (SM $\mathrm{oBR})$. The solubility parameters were calculated for the oligomers as well as for the butadiene rubber containing $100 \%$ of vinyl or cis/trans isomers, isoprene rubber, and the SSBR Buna SL 4525-0 based on its styrene content. The solubility parameters of the oligomers have very similar values to the parameters of the rubbers indicating good mutual miscibility. 
TABLE I

FORMULATIONS OF THE SAMPLE COMPOUNDS, IN PHR ${ }^{a}$

\begin{tabular}{lccccc}
\hline Compound & SSBR/BR & Silica & Modified silica & TESPD & Covering agent \\
\hline Reference & $75 / 25$ & 50 & - & - & - \\
R-coupling (in situ) & $75 / 25$ & 50 & - & 4 & - \\
R-covering (in situ) & $75 / 25$ & 50 & - & - & 11 \\
R-covering & $75 / 25$ & - & $53.7^{b}$ & - & - \\
SM oBR & $75 / 25$ & - & $57.1^{b}$ & - & - \\
SM cyclo & $75 / 25$ & - & $60.8^{b}$ & - & - \\
SM tert & $75 / 25$ & - & $62.5^{b}$ & - & - \\
SM 1-hexa & $75 / 25$ & - & $69.5^{b}$ & - & - \\
\hline
\end{tabular}

${ }^{a}$ Other ingredients: zinc oxide, 2.5 ; stearic acid, $2.5 ; \mathrm{N}$-(1,3-dimethylbutyl)- $N$-phenyl-p-phenylenediamine [6PPD], 2; polymerized 2,2,4-trimethyl-1,1-dihydroquinoline [TMQ], 2; treated distillate aromatic extract [TDAE], 15; sulfur, 1.4; 1,3diphenylguanidine [DPG], 2; $N$-tert-butyl-benzothiazole sulfonamide [TBBS], 1.7.

${ }^{b}$ Amounts of the modified silicas were calculated based on TGA results to keep equal $\mathrm{SiO}_{2}$ content, $50 \mathrm{phr}$.

For the study the following rubber samples were prepared (formulations in Table 1, mixing procedure in Table 2):

- Reference: filled with unmodified silica

- R-coupling (in situ): in situ silanized with TESPD

- R-covering (in situ): in situ silanized with dodecyltriethoxysilane (covering agent)

- R-covering: filled with silica pre-silanized with dodecyltriethoxysilane (similar conditions to the oligomer-grafted samples)

- SM oBR: filled with silica grafted with hydrogenated oligomer without attached thiols

- SM cyclo: filled with silica grafted with hydrogenated oligomer with cyclohexanethiol

TABLE II

MIXING PROCEDURE

Time

Action

Step 1

0.00

1.20

2.40

4.00

5.00

12.00

Step 2 (finalizing coupling reaction)
0.00
2.00
10.00

Step 3

0.00

1.30

3.00
Add rubber

Add $1 / 3$ filler, ${ }^{a} 1 / 2$ silane

Add $1 / 3$ filler, ${ }^{a} 1 / 2$ silane, TDAE

Add 1/3 filler, ${ }^{a}$ zinc oxide, stearic acid, 6PPD, TMQ Increase torque (increase temperature to $130{ }^{\circ} \mathrm{C}$ )

Stop mixing

Add elastomer pre-mix

Increase torque (increase temperature to $130{ }^{\circ} \mathrm{C}$ )

Stop mixing

Add elastomer pre-mix

Add all curatives (sulfur, DPG, TBBS)

Stop mixing

\footnotetext{
${ }^{a}$ The silica after modification was obtained in a loose powder form that required an unusually long mixing procedure.
} 


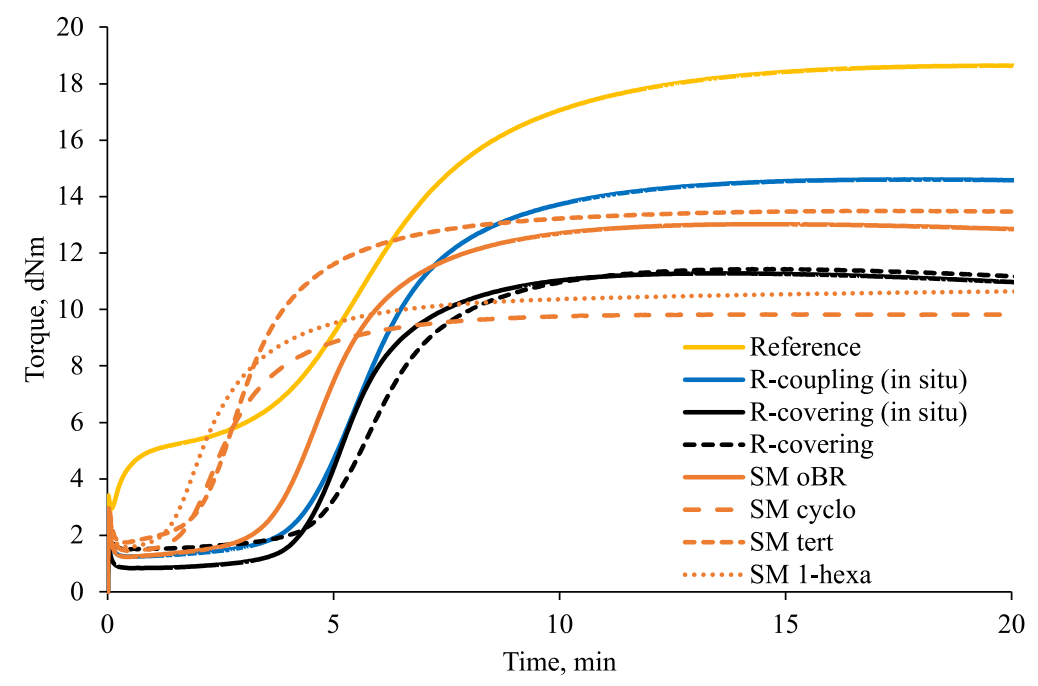

FIG. 2. - Kinetics of vulcanization curves of the rubber compounds.

- SM tert: filled with silica grafted with hydrogenated oligomer with tert-dodecanethiol

- SM 1-hexa: filled with silica grafted with hydrogenated oligomer with 1-hexadecanethiol

\section{RESULTS}

The kinetics of vulcanization results (Figure 2) reveal that the scorch time of the compounds filled with the oligomer-grafted silicas is significantly decreased. It is most likely caused by the remaining reactivity of the monosulfidic bonds present in the modified oligomers that recombine with curatives during vulcanization. This is especially visible in comparison with the SM oBR sample that contains oligomers which are non-modified with thiols, and thus its scorch time is significantly longer. The monosulfidic bonds can re-arrange with the curatives changing the crosslink structure by reaction with the rubber macromolecules or recombination of neighboring monosulfidic bonds (Figure 3). However, this does not influence the cross-link density significantly, which is comparable for all the samples, excluding the SM tert sample side-branched with tert-dodecanethiol (Figure 4). This suggests a prevalent reactivity of the monosulfidic bonds toward the rubber macromolecules instead of recombination (Figure 3A). It is evidenced by a higher increase of torque during the vulcanization kinetic test for the SM tert sample in comparison

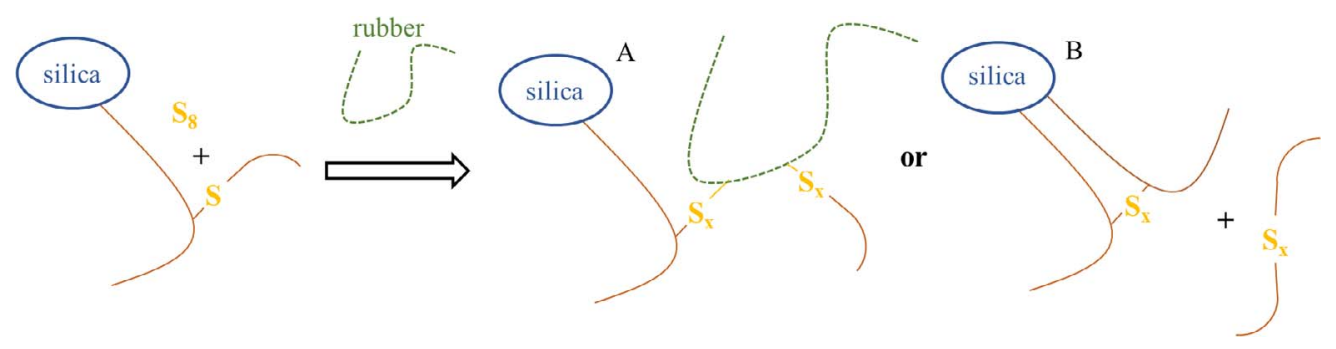

FIG. 3. - Possible reactions of monosulfidic bonds during sulfur vulcanization: (A) reaction with rubber; (B) recombination of two neighboring monosulfidic groups. 


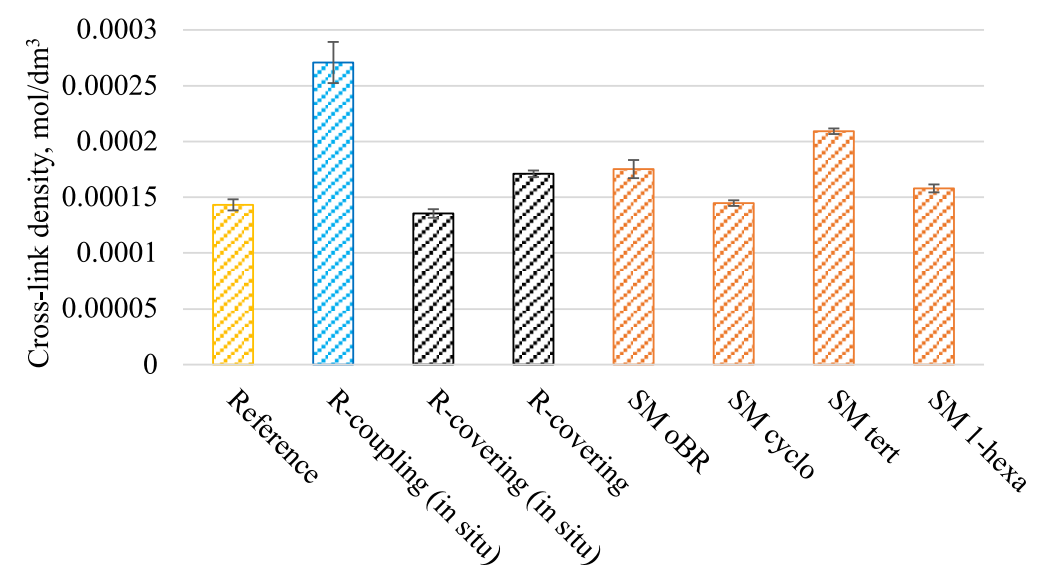

FIG. 4. - Cross-link density of the rubber vulcanizates.

with SM cyclo and SM 1-hexa. Also in the case of the sample containing in situ coupled silica, the covalent bonds between the silica and rubber produced by TESPD act as cross-links contributing to their increased number.

Owing to the fact that the monosulfidic groups of the oligomers are reactive during vulcanization, the Payne effect was measured for non-vulcanized compounds to exclude the possible effects from rubber-oligomer coupling (Figure 5). The lowest Payne effect and modulus at $100 \%$ of strain exhibit the sample containing in situ silanized silica with the covering agent. This shows that the pre-modification of the silica is not as effective as in situ silanization. Such effect is most likely caused by breaking down the silica agglomerates during mixing that uncovers new nonmodified sites on the silica surface. This contributes to higher filler-filler interactions visible in the Payne effect measurements. When the in situ silanized silica is compared with the pre-modified silica with the covering agent, the Payne effect value increases from 74 to $114 \mathrm{kPa}$. A similar effect can be expected when the pre-modified silicas with the oligomers are mixed, which is also visible in the Payne effect results. However, an intriguing phenomena is observed. The addition of the silica

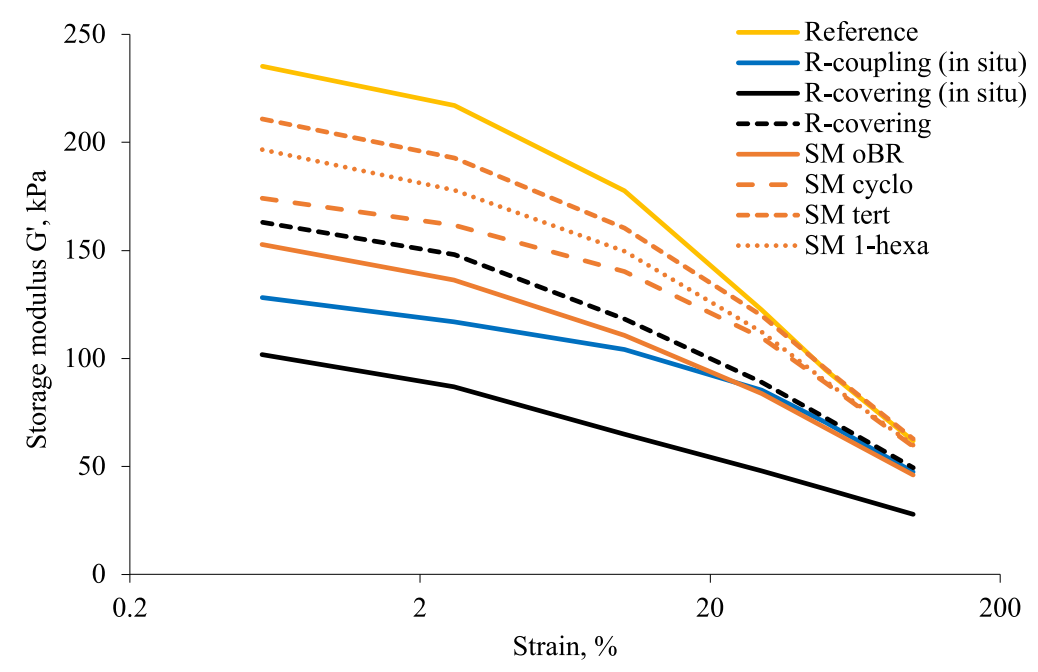

FIG. 5. — Payne effect of the rubber compounds (not vulcanized). 
TABLE III

PAYNe EFFECT $\left(\Delta G^{\prime}\right)$ AND MOdUlus AT $100 \%$ of STRAin $\left(G^{\prime} 100 \%\right)$ OF THE RUBBER COMPOUNDS

\begin{tabular}{lcc}
\hline Compound & $\Delta G^{\prime}, \mathrm{kPa}$ & $G^{\prime}{ }_{100 \%}, \mathrm{kPa}$ \\
\hline Reference & 173 & 62 \\
R-coupling (in situ) & 81 & 47 \\
R-covering (in situ) & 74 & 28 \\
R-covering & 114 & 49 \\
SM oBR & 107 & 46 \\
SM cyclo & 115 & 59 \\
SM tert & 148 & 63 \\
SM 1-hexa & 137 & 60 \\
\hline
\end{tabular}

modified with non-branched oligomer caused a slight decrease in Payne effect in comparison to the R-covering sample, most likely due to much larger molecular weight of the oligomer in comparison with the dodecyltriethoxysilane that can more effectively hydrophobize the silica surface. But when the silicas with branched oligomers are added, the Payne effect increases in comparison with the SM oBR sample. The branching of the oligomers with thiols took place in a second step of the silica modification, when the main chain of the oligomer was already grafted on the silica. Therefore, it is highly unlikely that more non-modified sites on the silica surface were uncovered during this sample mixing in comparison with SM oBR. Thus, this increase of Payne effect cannot be a result of higher filler-filler interactions.

There are several hypotheses explaining the nature of the Payne effect in filled rubber. Analyzing the Payne effect in rubber filled with the state-of-the-art silica-silane systems shows that the Payne effect comes from the strong filler-filler interactions. When a silane couples or covers the silica surface effectively the Payne effect value decreases significantly due to a significant reduction of the filler-filler interactions. However, grafting long oligomer chains on the silica surface changes the interphase properties by implementing macromolecular entanglements. In such circumstances the increase of the Payne effect for the compounds containing surface modified silica with the branched oligomers can be explained on the basis of Dannenberg's molecular slippage model. ${ }^{8}$ This model assumes that the Payne effect is a consequence of adsorption and desorption of rubber chains on the filler surface under oscillating strain. Here, it is postulated that the increase of the Payne effect for the compounds containing the branched-oligomer modified silicas stems from the formation of relatively strong oligomer-rubber entanglements. This shows that physical entanglements can contribute to improved silica-rubber interactions and therefore provide additional reinforcement. The advantage of this new coupling approach is its re-connectability-if the rubber-silica interphase is decoupled under an external strain it is still able to re-constitute after the strain disappears due to the physical nature of coupling and good rubber-oligomer miscibility.

The high oligomer-rubber interactions are also evidenced by the $G^{\prime}$ modulus value at $100 \%$ of strain (Table 3). The compounds containing silicas grafted with the branched oligomers exhibit the highest value of this parameter, next to the reference sample filled with non-modified silica. Although the $G^{\prime}{ }_{100 \%}$ values for these compounds are similar, they stem from different physical phenomena. For the samples containing the modified silicas, the high values of the modulus are a result of strong entangling between the branched oligomers and the rubber. Whereas the nonmodified silica exhibits high surface energy that might bind the rubber macromolecules by facilitating a high value of work of adhesion between the silica and rubber, ${ }^{9}$ possibly stemming from inducing local dipoles on rubber macromolecules that interact with the polar silica surface. 


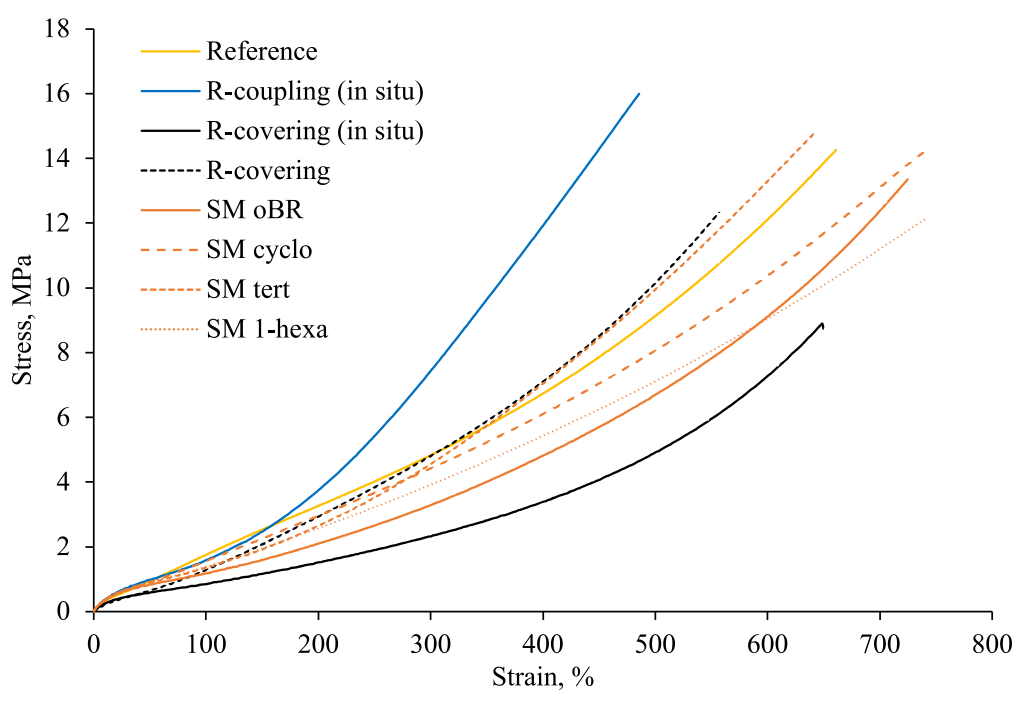

FIG. 6. - Stress-strain curves of the rubber vulcanizates.

Also, due to high filler-filler interactions of the silica particles, a considerable amount of occluded rubber is formed that could contribute to the hydrodynamic effect ${ }^{10}$ or be released at high strain, increasing the $G^{\prime}$ value. Branching of the oligomers results in a very significant improvement of the interactions with rubber in comparison with the non-branched oligomer. This is visible in the Payne effect but is more evident in the $G^{\prime}{ }_{100 \%}$ value, which for the SM oBR is on the same level as the sample containing pre-modified silica with the covering agent or the one with the in situ silanized silica with coupling agent.

Application of the oligomers as an alternative coupling agent results in a noticeable increase of elongation at break (Figure 6; Table 4) due to the presence of the grafted oligomers that contribute to increasing the amount of the polymeric phase. Even though the reactivity of the monosulfidic bonds in the presence of the sulfur curing system was shown, it is assumed that the oligomers do not take part in the cross-linking; therefore the amount of curatives was not adjusted for the additional amount of oligomer. This resulted in similar cross-link densities of all the rubber vulcanizates except of the samples containing TESPD or tert-dodecanethiol branched oligomer. In this view the tensile properties of the samples containing oligomer-modified silicas varies significantly. Application of the thiol-branched oligomers increased the modulus and tensile strength of the rubber, when cyclic or branched side chains were implemented (SM cyclo and SM tert-in this case it partially stems from the higher cross-link density), while the long linear branches finally decreased the tensile strength of rubber. However, below $600 \%$ of elongation the SM 1-hexa sample exhibits higher modulus than SM oBR. This shows that the molecular structure of the grafted oligomers plays a vital role in the rubber reinforcement because it influences the ability of an oligomer molecule to form reinforcing entanglements. The improvement of mechanical properties gained by formation of entanglements is also evidenced by comparison of the Velcro-modified silicas with the silicas treated by the covering agent. The vulcanizates filled with the Velcromodified silicas always exhibit higher tensile strength and elongation at break, which leaves room for further improvement by adjusting the curing system.

The mechanical properties of the rubber vulcanizates result from the rubber matrix properties, rubber-filler interactions, and the ability of a filler to form a reinforcing network. The unmodified silica reinforces the rubber by forming a strong filler-filler network connected via hydrogen bonds, 
TABLE IV

Tensile Strength (Ts), Elongation at Break (EB), Moduli at 100\%

(M100) OF STRAIN

\begin{tabular}{lccc}
\hline Compound & Ts, MPa & Eb, \% & M100 \\
\hline Reference & 14.1 & 643 & 1.8 \\
R-coupling (in situ) & 15.5 & 468 & 1.6 \\
R-covering (in situ) & 8.3 & 618 & 0.8 \\
R-covering & 11.7 & 551 & 1.3 \\
SM oBR & 13.5 & 705 & 1.2 \\
SM cyclo & 14.1 & 753 & 1.6 \\
SM tert & 14.2 & 633 & 1.4 \\
SM 1-hexa & 12.3 & 709 & 1.4 \\
\hline
\end{tabular}

whereas the coupling agent provides strong covalent silica-rubber bonds. When a covering agent is used, the mechanical properties deteriorate due to decreasing filler-filler interactions. The rubber vulcanizate containing pre-modified silica with the covering agent exhibits improved tensile strength in comparison with the one containing in situ covered silica (Figure 6; Table 4). This is in line with the Payne effect observations and results from the contribution of the filler network formed by the non-modified sites on the silica surface uncovered during mixing. The pre-modification of silica before compounding is not able to cover completely the surface of the silica clusters. This is because they are connected by strong polar interactions, which prevents silane molecules from diffusing and reacting with the strongly bonded surfaces. However, during mixing high shear forces separate the clusters and uncover unmodified areas of silica. This leads to higher filler-filler interactions, thus improving the reinforcing effect.

In Figure 7, the Payne effect is plotted against the vulcanizate tensile strength to show the nature of reinforcement that silica-based systems can provide. Non-modified silica shows high reinforcing potential originating from strong filler-filler interactions. High polarity of nonmodified silica results in reduction of its wettability by the rubber molecules; ${ }^{9}$ however, its reinforcing performance is encompassed by strong interactions between the polar particles that form a strong secondary structure resulting in high mechanical properties but poor dynamic and fatigue performance. ${ }^{11}$ Alternatively, the use of a coupling agent provides high filler-rubber interactions via strong covalent bonds. In the absence of one of these effects a significant drop in mechanical properties is observed (R-covering (in situ)). The application of a new coupling system that is based on physical entanglement formations between the rubber and oligomers grafted on the silica surface results in a significant reinforcing effect, without the need of covalent bonds. The entanglement formations are evidenced by higher tensile strength than provided by the covering agent. Moreover a lower Payne effect is obtained in comparison with the non-modified silica. Thus, the filler-filler interactions that allow better silica dispersion and simultaneously an effective coupling with rubber provided by physical entanglements are reduced.

To evaluate the potential Velcro-like behavior of the newly developed coupling system, a hysteresis test was performed followed by thermal conditioning of the vulcanizates. During the hysteresis test, five cycles of stretching were applied to reach $300 \%$ of elongation each time. Modulus at $300 \%$ of elongation (M300) measured for the fifth cycle shows a decrease in comparison with the first cycle due to a deterioration of both filler-filler and filler-rubber interactions. Afterward a thermal conditioning $\left(4 \mathrm{~h}\right.$ at $\left.80{ }^{\circ} \mathrm{C}\right)$ was performed to stimulate the reinforcing system to recover, and the mechanical test was repeated. Figure 8 displays the results showing a decrease in the moduli value between the first and fifth cycle and recovery after the 


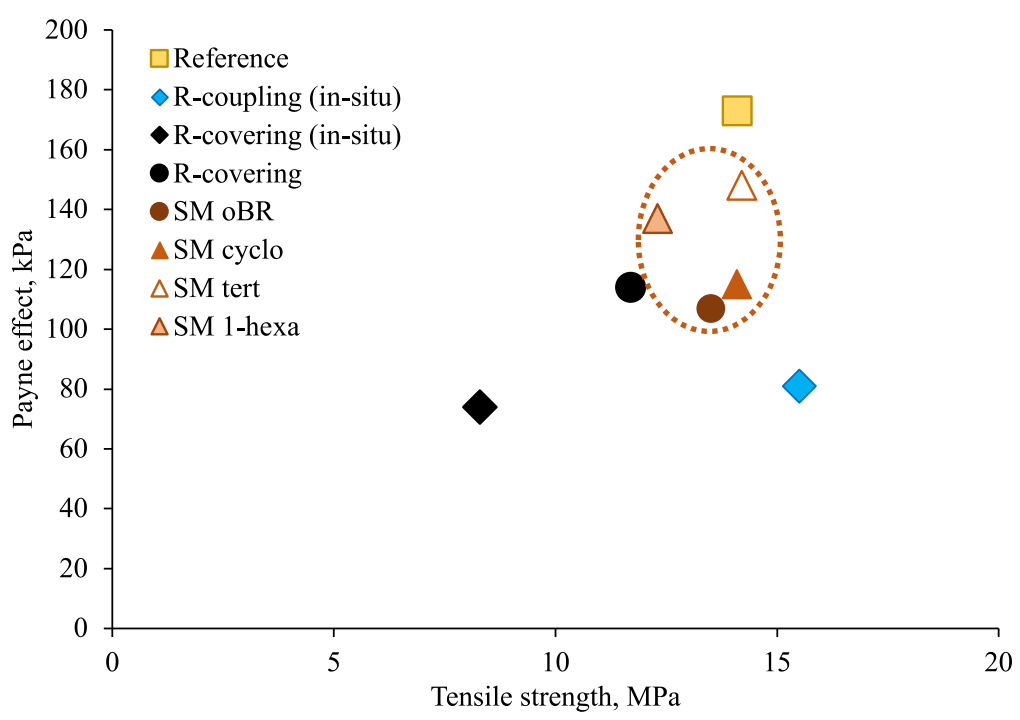

FIG. 7. — Payne effect vs tensile strength plot.

conditioning. The sample filled with silica chemically coupled to the rubber suffers from the highest decrease of M300, and the recovery is very limited. This probably stems from the non-reconnectable nature of the sulfur links formed between the silica and rubber, which once broken are unable to recombine during the thermal stimuli. The samples filled with non-treated or covered by short hydrocarbon chain silicas exhibit a relatively low decrease of the M300 between the first and fifth cycle and a noticeable recovery level. The low decrease is caused by the non-covalent character of the silica-rubber compatibilization and the contribution from filler-filler interactions that are partially restored in the cases of the Reference and R-covering samples. The most significant changes are visible in the behavior of the samples modified with the oligomer brushes, which show

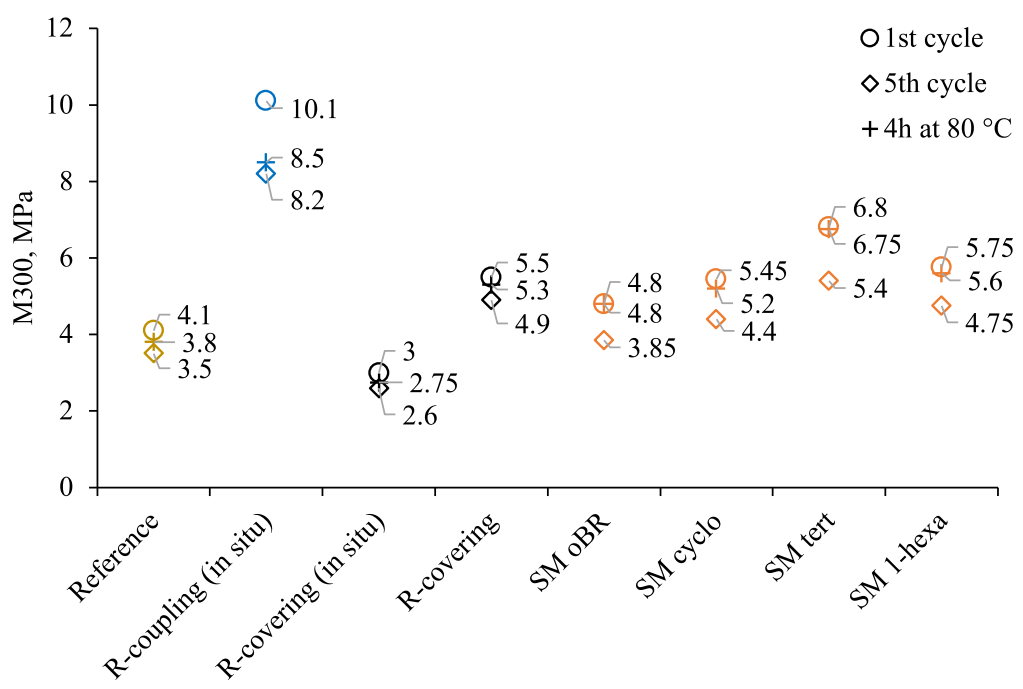

FIG. 8. - Moduli at $300 \%$ of elongation measured on first and fifth cycle of the hysteresis test and after $4 \mathrm{~h}$ thermal conditioning at $80{ }^{\circ} \mathrm{C}$. 
the highest drop in M300 between the first and fifth cycle due to a significant disentanglement effect. Physical entanglements require less energy to delaminate the silica-filler interphase than covalent bonds, thus the significant decrease of M300 is observed. However, after the thermal conditioning the mobile brushes grafted on the silica surface are able to re-establish the entanglement connections with the rubber phase, resulting in a significant recovery of M300, reaching even 100\% for SM oBR. This depicts the high re-connectability potential of the newly developed coupling system exhibiting Velcro-like behavior.

\section{CONCLUSIONS}

An alternative silica-rubber coupling system is presented that provides a different reinforcing mechanism based on strong entanglements between rubber and branched-oligomer chains grafted on the silica surface. The strong filler-rubber interactions (however, still lower than those of the state-of-the-art chemical coupling) are evidenced by high tensile strength of the rubber vulcanizates and high modulus value at $100 \%$ of strain achieved during the Payne effect measurement for the compounds. The spatial structure of the branched oligomer plays a significant role in the formation of entanglements, and thus it is vital for good mechanical properties. Branched or cyclic structures of the side chains of the oligomers are more beneficial in terms of rubber reinforcement than linear ones. Application of the branched oligomers for the silica coupling increases the Payne effect of the compounds. However, the increased Payne effect is not due to higher filler-filler interactions but due to the formation of entanglements with the rubber matrix that strongly influences the adsorption/desorption of the rubber molecules on the silica surface. Thus, the Payne effect results cannot be interpreted in the same way as for commonly used coupling/covering agents. The reconnectability of the silica-rubber interactions for the new system was shown by the significant recovery of the stress at $300 \%$ of elongation measured before and after hysteresis cycles and thermal conditioning. The developed coupling system is a promising alternative to currently used coupling agents and will be explored further.

\section{REFERENCES}

${ }^{1}$ W. H. Waddell and L. R. Evans, RubBer CHEM. TECHNOL. 69, 377 (1996).

${ }^{2}$ T. Stephens, "How a Swiss invention hooked the world," January 4, 2007, http://www.swissinfo.ch/eng/ velcro_how-a-swiss-invention-hooked-the-world/5653568. Accessed date November 29, 2020.

${ }^{3}$ Y. Ahn, Y. Jang, N. Selvapalam, G. Yun, and K. Kim, Angew. Chem. Int. Ed. 52, 3140 (2013).

${ }^{4}$ M. Sievers, J. C. Namyslo, F. Lederle, and E. G. Hubner, Express Polym. Lett. 12, 556 (2018).

${ }^{5}$ K. P. O'Connor and T. C. B. McLeish, Macromolecules 26, 7322 (1993).

${ }^{6}$ T. Gutsmann, T. Hassenkam, J. A. Cutroni, and P. K. Hansma, Biophys. J. 89, 536 (2005).

${ }^{7}$ R. Anyszka, W. Dierkes, D. M. Bielinski, E. Sarlin, and A. Blume, "Mimicking the Velcro System from Nature for an Alternative Rubber/Silica Coupling System,” presented at the KHK 13th Rubber Fall Colloquium, Hannover, Germany, November 6-8, 2018.

${ }^{8}$ E. M. Dannenberg, RubBer Chem. TeChNOL. 48, 410, (1975).

${ }^{9}$ K. W. Stöckelhuber, A. S. Svistkov, A. G. Pelevin, and G. Heinrich, Macromolecules 44, 4366 (2011).

${ }^{10}$ J. Jin, "Influence of Compounding and Mixing on Filler Dispersion and Curing Behavior of Silica Compounds," Ph.D.

Dissertation, University of Twente, 2020.

${ }^{11}$ H. Yao, G. Weng, Y. Liu, K. Fu, A. Chang, and Z-R. Chen, J. Appl. Polym. Sci. 132, 41980 (2015). 